\title{
Correction to: The effectiveness of a life style modification and peer support home blood pressure monitoring in control of hypertension: protocol for a cluster randomized controlled trial
}

Tin Tin Su ${ }^{1 *}$, Hazreen Abdul Majid ${ }^{1}$, Azmi Mohamed Naharr ${ }^{2}$, Nurul Ain Azizan ${ }^{1}$, Farizah Mohd Hairi ${ }^{1}$, Nithiah Thangiah", Maznah Dahlui ${ }^{1}$, Awang Bulgiba ${ }^{3}$ and Liam J. Murray ${ }^{4}$

\section{Correction}

After publication of the article [1], it has been brought to our attention that the methodology outlined in the original article was not able to be fully carried out. The article planned a two armed randomized control trial. However, due to a lower response than expected and one housing complex dropping out from the study, the method was changed to pre- and post-intervention with no control group. All other methods were conducted as outlined in the original article.

\footnotetext{
Author details

${ }^{1}$ Centre for Population Health (CePH), Department of Social and Preventive Medicine, Faculty of Medicine, University of Malaya, Kuala Lumpur, Malaysia. ${ }^{2}$ Department of Sports Medicine, Faculty of Medicine, University of Malaya, Kuala Lumpur, Malaysia. ${ }^{3}$ Julius Centre University of Malaya (JCUM), Department of Social and Preventive Medicine, Faculty of Medicine, University of Malaya, Kuala Lumpur, Malaysia. ${ }^{4}$ Centre for Public Health, Queen's University of Belfast, Belfast, Ireland.
}

Received: 13 October 2017 Accepted: 16 October 2017

Published online: 06 November 2017

\section{Reference}

1. Su T, Majid H, Nahar A, Azizan N, Hairi F, Thangiah N, et al. The effectiveness of a life style modification and peer support home blood pressure monitoring in control of hypertension: protocol for a cluster randomized controlled trial. BMC Public Health. 2014;14(Suppl 3):S4. doi: 10.1186/14712458-14-S3-S4

\footnotetext{
* Correspondence: tintinsu03@yahoo.com

'Centre for Population Health (CePH), Department of Social and Preventive Medicine, Faculty of Medicine, University of Malaya, Kuala Lumpur, Malaysia
} 